\title{
Guiding Corpus-based Set Expansion by Auxiliary Sets Generation and Co-Expansion
}

\author{
Jiaxin Huang ${ }^{1 *}$, Yiqing Xie ${ }^{2 *}$, Yu Meng ${ }^{1}$, Jiaming Shen ${ }^{1}$, Yunyi Zhang ${ }^{1}$, Jiawei Han ${ }^{1}$ \\ ${ }^{1}$ University of Illinois at Urbana-Champaign, IL, USA \\ ${ }^{2}$ The Hong Kong University of Science and Technology, Hong Kong, China \\ ${ }^{1}\left\{\right.$ jiaxinh3, yumeng5, js2, yzhan238, hanj\}@illinois.edu ${ }^{2}$ yxieal@ust.hk
}

\begin{abstract}
Given a small set of seed entities (e.g., "USA", "Russia"), corpusbased set expansion is to induce an extensive set of entities which share the same semantic class (Country in this example) from a given corpus. Set expansion benefits a wide range of downstream applications in knowledge discovery, such as web search, taxonomy construction, and query suggestion. Existing corpus-based set expansion algorithms typically bootstrap the given seeds by incorporating lexical patterns and distributional similarity. However, due to no negative sets provided explicitly, these methods suffer from semantic drift caused by expanding the seed set freely without guidance. We propose a new framework, Set-CoExpan, that automatically generates auxiliary sets as negative sets that are closely related to the target set of user's interest, and then performs multiple sets co-expansion that extracts discriminative features by comparing target set with auxiliary sets, to form multiple cohesive sets that are distinctive from one another, thus resolving the semantic drift issue. In this paper we demonstrate that by generating auxiliary sets, we can guide the expansion process of target set to avoid touching those ambiguous areas around the border with auxiliary sets, and we show that Set-CoExpan outperforms strong baseline methods significantly.
\end{abstract}

\section{CCS CONCEPTS}

- Information systems $\rightarrow$ Data mining; • Computing methodologies $\rightarrow$ Lexical semantics;

\section{KEYWORDS}

Set Expansion, Bootstrap Methods, Semantic Computing, Web Mining

\section{ACM Reference Format:}

Jiaxin Huang ${ }^{1 *}$, Yiqing $\mathrm{Xie}^{2 *}$, Yu Meng ${ }^{1}$, Jiaming Shen ${ }^{1}$, Yunyi Zhang ${ }^{1}$, Jiawei $\mathrm{Han}^{1}$. 2020. Guiding Corpus-based Set Expansion by Auxiliary Sets Generation and Co-Expansion. In Proceedings of The Web Conference 2020 (WWW'20), April 20-24, 2020, Taipei, Taiwan. ACM, New York, NY, USA, 11 pages. https://doi.org/10.1145/3366423.3380284

\footnotetext{
${ }^{*}$ Equal Contribution
}

This paper is published under the Creative Commons Attribution 4.0 International (CC BY 4.0) license. Authors reserve their rights to disseminate the work on their personal and corporate Web sites with the appropriate attribution.

WWW'20, April 20-24, 2020, Taipei, Taiwan

(C) 2020 IW3C2 (International World Wide Web Conference Committee), published under Creative Commons CC BY 4.0 License.

ACM ISBN 978-1-4503-7023-3/20/04.

https://doi.org/10.1145/3366423.3380284

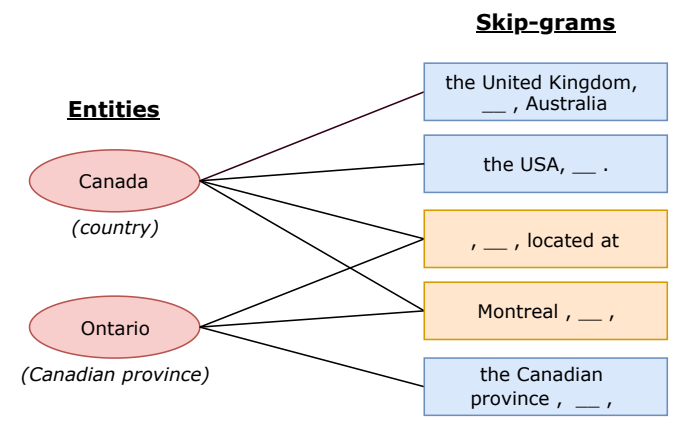

Figure 1: Examples of shared skip-grams between country and province/states entities.

\section{INTRODUCTION}

The task of set expansion is to enrich the set of given seeds belonging to a certain semantic class (e.g., given "United States", "Canada", and "China", set expansion algorithms would extend the set by terms such as "France", "Germany" and "Australia", which also belong to the semantic class of Country). Set expansion benefits a variety of downstream applications in natural language processing and knowledge discovery, such as web search [5], query suggestion [4], question answering [16, 24], and taxonomy construction [22].

Existing set expansion methods that rely on a large corpus typically bootstrap the initial seeds by refining the context feature pool and candidate term pool in an iterative manner. Though achieving reasonably good results, they still suffer from the problems of semantic drifting and entity intrusion in the expansion process. Typical errors come from closely related entities of different granularity or semantic types. Consider the example in Figure 1, we draw a bipartite graph where entities are linked to local contexts they appear in. Despite the difference in granularity of "Canada" as a country and "Ontario" as a Canadian province, they share some local contexts. These shared contexts, when introduced into the context feature pool, result in cross-category expansion and harm the final output continuously in the iterative process. Similarly, the shared context may bring in alien semantic types in set expansion. For example, a disease "lung cancer" may bring in symptom "chest pain" since both may share some common local contexts, such as "suffer from __." Such kind of semantic drift is caused by the uncontrolled expansion without guidance. Since there are no negative sets explicitly given, and either initial seed or generated new members could be at the border of multiple sets, uncontrolled expansion will likely cross the border and generate off-class entities.

Some previous studies have explored to incorporate external knowledge in set expansion using either implicit supervision from 
other queries or human given negative examples. $[11,20]$ use other queries in the dataset to provide mutual exclusive signals, relying on the assumption that different queries belong to different semantic classes, which does not necessarily hold when other queries are not visible. [10] utilizes human given negative examples to constrain the scope of set expansion. However, an implicit assumption is that possible directions of error need to be predicted in advance, and human effort is needed to provide cases for each direction. The applicability of these methods is hindered by their prerequisites as they are not adaptable to specialized domains with very few experts.

To tackle the problem of semantic drift in set expansion, we explore a fully automated approach without negative sets or assumptions explicitly provided by human experts. We observe that a typical source of error comes from the entities from different semantic classes that may share some common relations to the target class. Word embedding has shown effective in capturing certain relations between entities, such as the parallelism of $v$ (Berlin) $-v$ (Germany) and $v$ (Paris $)-v($ France $)$ in the embedding space, which can also be used to distinguish City from Country. If one can recognize such subtle relationships between entities belonging to different semantic classes, one can capture such subtlety and use it to separate entities from different classes and conduct set co-expansion. Such co-expansion may incorporate signals from all the related, participating classes, keep warning the target class not to cross over the boundaries of its possible rivals, and guide the expanding direction of each set by avoiding to bump into each other's territory. The co-expansion of such multiple rival sets benefits each other from mutually exclusive signals, and the quality of multiple sets expansion can be improved simultaneously.

We propose a framework called Set-CoExpan with its workflow shown in Figure 2. It consists of two modules that are operated iteratively: Auxiliary sets generation module that finds auxiliary sets holding certain relations with the target set in an unsupervised way, and multiple sets co-expansion module that takes multiple sets as input and extracts the most discriminative features to tell the target class from auxiliary sets. The auxiliary sets generation module first retrieves semantically related terms to each seed element in an embedding space that captures topical similarity. These related terms are then grouped by their semantic types, captured unsupervisedly by intra-seed clustering and inter-seed merging. The latter step captures parallel relations among inter-seed terms by imposing a parallelogram in the embedding space. The second module, multiple sets co-expansion, takes the target seed set as well as auxiliary sets as input. By incorporating knowledge from both seed set and auxiliary sets, we can control the expanding directions of multiple sets. Specifically, context features are scored by how well they can tell different sets apart, and the algorithm drives the expanding direction away from ambiguous areas.

We demonstrate the effectiveness of our framework through a series of experiments on two real-world datasets, and show that Set-CoExpan outperforms strong baselines significantly. We also provide examples and qualitative analysis of auxiliary sets to showcase the effectiveness and generalizability of the proposed approach.

Our contribution can be summarized as follows: (1) An auxiliary sets generation module that extracts entities that share certain relations to thus different from the target semantic class. (2) A multiple sets co-expansion module that utilizes the mutual exclusive signals to extract features with the most discriminative power to expand multiple sets simultaneously. (3) Comprehensive experiments and case studies that prove the effectiveness of Set-CoExpan and justify its generalizability.

\section{RELATED WORK}

Web-based Set Expansion. Most of early set expansion methods are developed in online web applications. They typically submit a query consisting of seed entities to a web search engine and extract entities from retrieved web pages. Google Sets [21], now discontinued, uses latent semantic indexing technique to web pages for calculating word similarities. SEAL [24] extracts item lists from retrieved pages and constructs a heterogeneous network on which a graph-based ranking model is applied to rank entities. Lyretail [5] develops a supervised page-specific extractor to extract long-tail entities from the web. All these methods require an external search engine for online data collection, which is quite time-consuming. Our paper does not focus on this type of setting that uses retrieved documents as information resource. Instead, we address corpusbased set expansion problems and the related work is introduced below.

Corpus-based Set Expansion using Single Query. Many corpusbased set expansion methods [7-9, 13, 19, 27] are developed to bootstrap a single seed set query by iteratively refining a context feature pool and a candidate entity pool. Given the context feature pool, these methods build the candidate entity pool by adding only entities that co-occur frequently with high-quality context features. Meanwhile, they refine the context feature pool by including only those features which are commonly shared by entities in the expanded set. Based on this philosophy, SetExpan [19] develops a context feature selection module to select quality skip-gram features and designs a rank ensemble module to select quality entities. Similarly, SetExpander [13] captures distributional similarity on five different context types and learns a classifier to combine multiple contexts using an additional labeled dataset. Some corpus-based set expansion methods are designed to focus on one particular aspect of expansion process. For example, CaSE [26] pursues the expansion efficiency and thus develops a one-time ranking scheme based on a scoring function that incorporates both lexical patterns and distributional similarity. EgoSet [18] and FUSE [27] targets the multi-faceted expansion which allows for each seed term to generate multiple sets belonging to different senses. Atzori et al., [2] focus on singleton expansion (i.e., only one seed in the initial seed set).

Above methods only pay attention to the entities in the userinput seed query, suffering from possible semantic drift to other related classes while expanding the seed set. Vyas and Pantel [23] propose a human-in-the-loop set refinement method that enables human annotators to identify wrongly expanded entities in each iteration and then removes those context features shared by both positive and negative entities. SetExpander [13] implements a system that allows users to validate the correctness of expanded entities and do re-expansion at each iteration. These methods require additional human efforts and thus are not directly comparable to our method. 


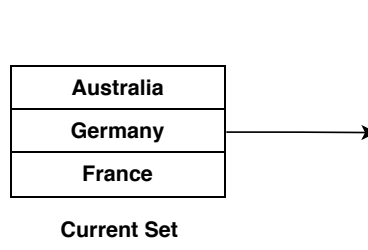

\begin{tabular}{|c|c|c|}
\hline Australia & Germany & France \\
\hline Brisbane & Stuttgart & Toulouse \\
\hline Canberra & Hamberg & Lyon \\
\hline Perth & Saxony & Rennes \\
\hline Queensland & Bavaria & Paris \\
\hline NSW & West Germany & Thomson Inc. \\
\hline
\end{tabular}

(1) Generating Auxiliary Sets
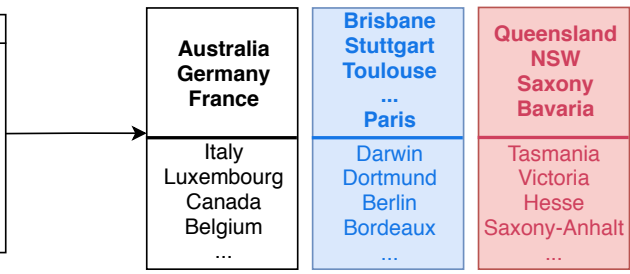

(2) Multiple sets Co-expansion

Figure 2: Workflow of Set-CoExpan in one iteration: For user-input country names, related terms such as provinces and cities are retrieved and clustered into auxiliary sets. Multiple sets are then co-expanded by extracting discriminative context features.

Corpus-based Set Expansion using Hybrid Query. Other methods proposed to use multiple different semantic classes as negative signals to constrain the scope of sets to be expanded. Basilisk [20] expands six categories given in the dataset simultaneously to avoid claiming terms in multiple categories. NOMEN [11] also allows for expanding multiple semantic classes simultaneously, and treat seeds from other classes as negative examples. These methods rely on other implicit queries in the dataset, which may not be relevant enough to provide mutual exclusive signals, and other queries may not even exist on every occasion. What's more, the assumption that different queries belong to different semantic classes may not even holds. [10] proposes an inference-based algorithm that leverages human-given negative examples to define the semantic class. For example, human can provide the expansion of female tennis players with male tennis players as negative signals. However, the method requires prior knowledge of possible errors among multiple dimensions. E.g., female football players and female volleyball players can also serve as negative examples and the list is never exhausted.

These refining methods basically proves the idea that expanding multiple semantic classes simultaneously leads to better results, since mutual exclusive signals can help distinguish those ambiguous terms. However, they all rely on external supervision from other classes provided by human, thus are not comparable to our method.

\section{PROBLEM DEFINITION}

In this section we give the definition of the task of corpus-based set expansion. The inputs are a collection of documents $\mathcal{D}=$ $\left\{d_{1}, d_{2}, \ldots, d_{|\mathcal{D}|}\right\}$ and a seed set $S$ provided by user. The elements in $S$ are seed terms that belong to the same target semantic class $C_{T}$, which is a superset of $S$. The output is a ranking list of entities which is computed based on the lexical context features and distributed representations learned from the given corpus.

In our method, we will not only look into the given set of seeds $S$, but also try to extract other seeds forming multiple auxiliary sets $C_{\text {aux }}=\left\{S_{1} \subsetneq C_{1}, S_{2} \subsetneq C_{2}, S_{3} \subsetneq C_{3}, \ldots, S_{|P|} \subsetneq C_{m}\right\}$ as rival sets that are closely related to but different from the target semantic class $C_{t}$, to guide the expanding process by incorporating the mutual exclusive knowledge from target and multiple auxiliary sets. Our method automatically generates multiple auxiliary sets and does not require any human effort.

\section{METHODOLOGY}

In this section, we introduce our proposed method by first giving an overview in Section 4.1 and then describing the details of two modules in Section 4.2 and 4.3.

\subsection{Method Overview}

For a given set of query entities, we gradually expand the seed set by adding a fixed number of new entities at each iteration. Two modules collaborate with each other in an iterative manner: Auxiliary Sets Generation and Multiple Sets Co-Expansion.

The first module is pictured in Figure 3. For all seeds in the target set, we automatically generate auxiliary sets as rival classes, and auxiliary set entities share the same relations with the target set. In this figure, the user wants to expand country names, and Set-CoExpan can extract entities that share certain relations with given seeds (e.g., Brisbane as a city located in Australia as a country, and Merkel as the prime minister of Germany as a country), and thus forming auxiliary sets of "Cities", "Provinces" and "Presidents/Prime Ministers". These new semantic classes benefit the model in distinguishing different granularities of the same concept or different semantic types.

In the second module, given target seed set and its auxiliary sets, we design a multiple sets co-expansion algorithm to let all the sets expand simultaneously in recognition of the existence of other sets, and treat elements in other sets as pitfalls to be avoided. Specifically, we use a context dependent similarity measure to describe the similarity of pairs of entities. We then extract the context features that maximize the similarity of pairs of elements from the same set, and minimize the similarity of pairs of elements from different sets. In this way, we can guide the set expansion process to avoid touching the ambiguous skip-grams shared by different semantic classes, then the target set and auxiliary sets can gradually expand their territories without grabbing off-class entities.

\subsection{Auxiliary Sets Generation}

As shown in Figure 3, in order to generate auxiliary sets that contain entities closely related to but different from the target semantic class, we aim to find entities forming groups that hold certain relations with given seed entities. For example, "Hamburg" and "Stuttgart" can form a small group in the embedding space due to their proximity in semantics. Another small group consists of "Brisbane" and "Canberra", and both groups share a parallel relation with seeds "Germany" and "Australia", represented as

$$
\text { Relation }\left(e_{1} \in C_{T}, g_{1}\right) \approx \operatorname{Relation}\left(e_{2} \in C_{T}, g_{2}\right)
$$

Using the information that $e_{1}$ and $e_{2}$ belong to the same semantic type, we can infer that two groups $g_{1}$ and $g_{2}$ are of the same semantic type and merge them into a cross-seed group. It is obvious to see that these cross-seed groups ensure consistent relations with the seed elements in the target semantic class, so that they can serve as auxiliary sets that we aim to look for. 


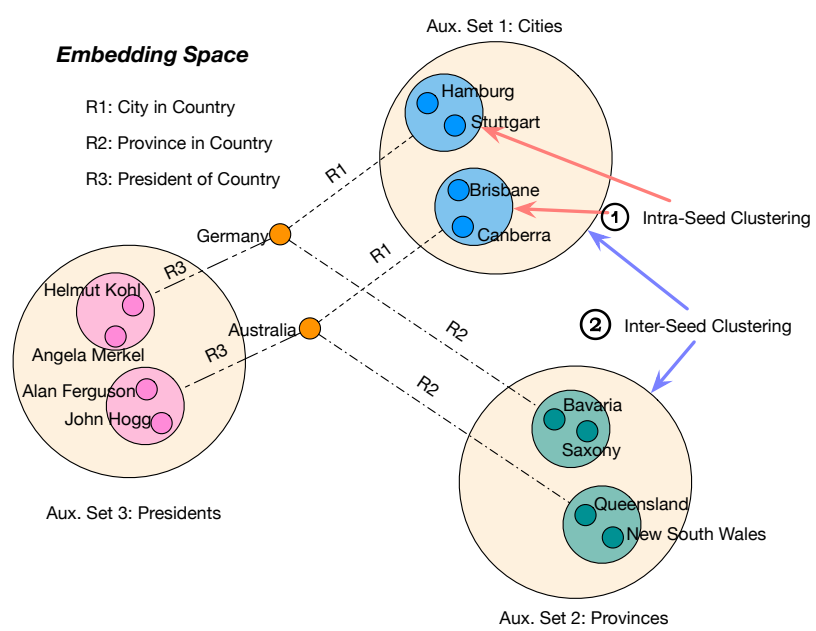

Figure 3: Auxiliary sets generation: Related terms of each seed are first clustered according to their semantic types; Cross-seed auxiliary sets are formed by capturing parallel offsets in the embedding space.

In the following we will introduce how we first retrieve pools of related words to seed elements, and then perform intra-seed and inter-seed clustering to form these auxiliary sets.

4.2.1 Semantic Learning and Related Terms Retrieval for Seed Elements. Word2Vec [15] has achieved wide success in capturing word semantics. To retrieve relevant terms to a certain seed, it is common practice to search for its nearest embedding space neighbors. Word2Vec assumes semantically similar words have similar local context (words in a fixed window size around the center word), and its corresponding training objective is

$$
\begin{aligned}
L_{l} & =\sum_{d \in D} \sum_{1 \leq i \leq|d|} \sum_{0<|j-i| \leq h} \log P\left(w_{j} \mid w_{i}\right) \\
& =\sum_{d \in D} \sum_{1 \leq i \leq|d|} \sum_{0<|j-i| \leq h} \log \frac{\exp \left(v_{w_{i}} u_{w_{j}}\right)}{\sum_{w_{j^{\prime}} \in V} \exp \left(v_{w_{i}} u_{w_{j^{\prime}}}\right)}
\end{aligned}
$$

where $h$ is the window size. A recent study [14] shows that documentlevel co-occurrences of words (i.e., global contexts) can capture topical relatedness between words, with the assumption that words appearing in similar documents tend to be topically similar. Here, we employ the same global context loss as in [14]:

$$
\begin{aligned}
L_{g} & =\sum_{d \in D} \sum_{1 \leq i \leq|d|} \log P\left(d \mid w_{i}\right) \\
& =\sum_{d \in D} \sum_{1 \leq i \leq|d|} \log \frac{\exp \left(v_{w_{i}} u_{d}\right)}{\sum_{d^{\prime} \in D} \exp \left(v_{w_{i}} u_{d^{\prime}}\right)}
\end{aligned}
$$

Global contexts characterize document-level co-occurrence statistics, while local contexts capture word-level co-occurrence statistics within local context windows. To encode the information of both sets of contexts into word embeddings, we propose an overall training objective with a weighted sum of global and local context losses.

$$
L=L_{l}+\lambda L_{g}
$$

After the embedding training is finished, for each entity $e$, we use cosine similarity to retrieve the $k$ most related terms, referred as $r_{e}$, from the vocabulary.
4.2.2 Cross-Seed Parallel Relations Clustering. Figure 3 shows an example of how auxiliary sets are confirmed as different semantic classes by the constraint of cross-seed parallel relations. Specifically, for a seed country "Australia”, its related words $r_{\text {Australia }}$ can first be clustered into small initial groups of the same semantic type: $G_{\text {Australia }}=\left\{g_{\text {Australia }}^{1}, g_{\text {Australia }}^{2}\right\}$, where $g_{\text {Australia }}^{1}=$ $\{$ Brisbane, Canberra, Perth $\}$ and $g_{\text {Australia }}^{2}=\{$ Queensland, NSW $\}$. For other seed elements like "Germany", several groups can also be clustered to form $G_{\text {Germany. Then inter-seed clustering can }}$ be performed to merge $g_{\text {Australia }}^{1}$ and $g_{\text {Germany }}^{1}$ into an auxiliary set since they hold parallel relations(City in Country) with corresponding seed element.

We perform two steps in extracting these auxiliary sets: (1) Intraseed clustering that clusters terms in each $r_{e}$ into initial groups of the same semantic class $g_{e}^{i}$ in a high precision. (2) Inter-seed clustering that merges initial groups that share same relations with corresponding elements into auxiliary sets.

In the first step, we cluster the retrieved related words in $r_{e}$ by their proximity in the embedding space. Since we obviously do not have a prior knowledge of the number of classes, and some outlier terms might not even be included in any high quality clusters, we apply Hierarchical Agglomerative Clustering (HAC) [17], a bottom-up hierarchical clustering method. The original result of HAC on $n$ data points is a binary tree-based representation named dendrogram with $n$ leaf nodes and $n-1$ internal nodes. A problem of directly applying this clustering would be the level chosen for splitting the data points. As groups of terms would be mixed with different semantic classes if the level chosen is too high, or missing meaningful groups if the level chosen is too low. To solve this issue, we insert user input seed set $S$ into the terms to be clustered, and the philosophy is that we stop the merging process when a seed element from target class is about to be merged with element related words. In this way, we make sure that the new clusters generated are of different semantic class from the original seed entities that belong to $C_{T}$.

In this HAC, our similarity measure uses the Euclidean distance between terms in the BERT [6] embedding space, where the representation of each entity is calculated by averaging the contextualized embedding of each occurrence in the corpus. We choose "complete" linkage for constructing more compact clusters.

In the second step, we use inter-seed clustering to capture parallel relations in Equation (1), and we interpret the Relation function as linear operations. Word analogy examples in [15] shows the Word2Vec embedding space hold the property of linearly capturing relations between words, so that $v($ Berlin $)-v($ Germany $) \approx$ $v($ Paris $)-v($ France $)$, with recent theoretical explanation in [1]. Studies on relation extraction $[3,12,25]$ relies on the idea that for a triplet of head <entity, relation, tail entity $>$ and their vector representation $\langle h, r, t\rangle$, the linear relation holds that $h+r \approx t$.

We also apply the above idea to capture cross-seed parallel relations. Specifically, we select the $i^{t h}$ initial group of element $e$, denoted as $g_{e}^{i}$. We then consider whether it can be merged with $g_{e^{\prime}}^{j}$ of a different seed element. To enlarge $g_{e}^{i}$ to cover more terms of the same type, we first expand $g_{e}^{i}$ into a larger group $g_{e, \text { expanded }}^{i}$ by a single query version of our set expansion algorithm later introduced in section 4.3. To represent the center of this expanded 
group, we take the average over all elements in $g_{e, \text { expanded }}^{i}$.

$$
v_{e, \text { expanded }}^{i}=\frac{\sum_{w \in g_{e, \text { expanded }}^{i}} v_{w}}{\left|g_{e, \text { expanded }}^{i}\right|}
$$

After that, we perform a parallel translation for $g_{e}^{i}$ from element $e$ to $e^{\prime}$ and obtain a pseudo center point for the new element $e^{\prime}$,

$$
v_{e \rightarrow e^{\prime}}^{i}=v_{e^{\prime}}-v_{e}+v_{e, \text { expanded }}^{i}
$$

We then retrieve 15 nearest neighbors from $v_{e^{\prime}}$ as the pseudo transitioned group $g_{e \rightarrow e^{\prime}}^{i}$ We then try to find an initial group $g_{e^{\prime}}^{j}$ of element $e^{\prime}$ that satisfies

$$
g_{e \rightarrow e^{\prime}}^{i} \cap g_{e^{\prime}}^{j}>\eta
$$

where $\eta$ is the merging threshold that determines whether two groups have enough overlapping terms. After conducting this merging process, we remove those unmerged initial groups to ensure that each remaining cluster ensures strong relations with seed elements by owning initial groups that share parallel cross-seed relations. We then treat each of these larger groups as an auxiliary set to distinguish our target seed set from. Algorithm 1 outlines this whole process of parallel relations clustering.

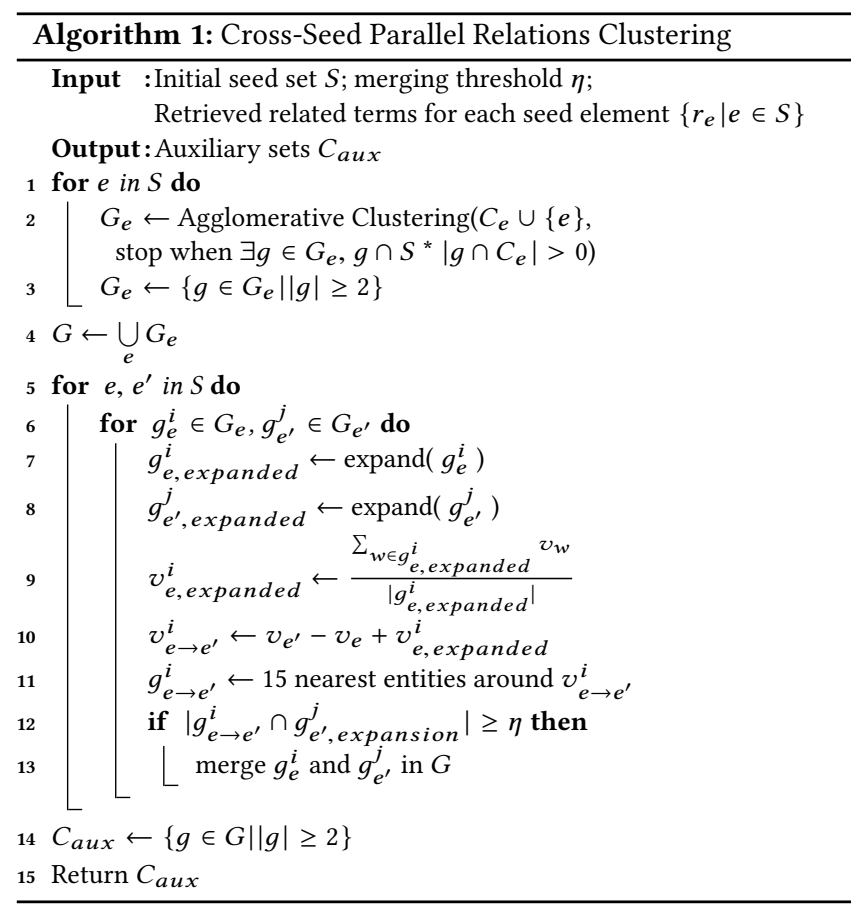

\subsection{Multiple Sets Co-Expansion}

4.3.1 Overview of the module. We iteratively refine two key elements in set expansion: feature pool and candidate pool. Feature pool stores common context features of seed entities, which best describe the target semantic class. Candidate pool stores the possible candidate entities to be expanded, and they are narrowed down by co-occurrence with features in the feature pool.

In each iteration, candidate terms that are most similar with the current expanded set are expanded. When more entities are added into the seed set, the feature pool will be refined by re-selecting the context features that maximize the similarity of user given and currently expanded terms.

We use skip-grams (e.g., $w_{-2} w_{-1} w_{1} w_{2}$ ) as local context features that describes an entity mention, and forms a bipartite graph as shown in Figure 1. To derive the weight of the edge connecting an entity with a skip-gram context, we first create a frequency matrix $\Phi_{e, c} \in \mathbb{R}^{N \times M}$ that counts their co-occurrence, where $N$ and $M$ are the number of candidate entities and skip-grams respectively. Then we define the weight connecting entity $e$ and context $c$ as

$$
f_{e, c}=\log \left(1+\Phi_{e, c}\right)\left[\frac{\log |N|}{\log \Phi_{e^{\prime}, c}}\right]
$$

which is the TF-IDF transformation [18]. An entity $e$ can thus be represented by an $M$-dim vector to derive similarity with other entities. However, such representation harms set expansion in two aspects: (1) the dimension of $M$ is obviously large and even if we only consider non-zero terms in seed elements, that dimension still grows as the size of expanded set grows. (2) Not all local contexts are type-indicative even if they do have a strong connection with a single seed entity. The weak supervision of given seed elements belonging to the same type can provide valuable information that the shared skip-grams among elements are more type-indicative. Therefore, context dependent similarity benefits set expansion tasks in that it only captures the type-indicative features of entities.

We adopt the context dependent similarity function $\operatorname{Sim}\left(e_{i}, e_{j} \mid F\right)$ defined in [19] using the weighted Jaccard similarity measure:

$$
\operatorname{Sim}\left(e_{1}, e_{2} \mid F\right)=\frac{\sum_{f \in F} \min \left(f_{e_{1}, c}, f_{e_{2}, c}\right)}{\sum_{f \in F} \max \left(f_{e_{1}, c}, f_{e_{2}, c}\right)}
$$

so that the selected features serve as references to compute the similarity of two entities.

4.3.2 Capturing Contrastive Skip-gram Contexts for Multiple Sets Co-Expansion. Let us consider the skip-gram context "the United Kingdom, __, Australia" in Figure 1, it can be connected to many country entities in the corpus, while very unlikely to be assigned to any other types of entities like provinces or cities. Thus, this kind of skip-grams are discriminative enough in indicating the type of entities. Nevertheless, if we look at the skip-gram "Montreal, __, , it is connected by both "Canada" as a country and "Ontario" as a Canadian province. The ambiguity of this skip-gram limits its power to discriminate upon different granularities of locations, thus is not helpful in distinguishing related but different sets, and may even cause semantic drift in later iterations.

In order to capture contrastive skip-gram contexts for multiple sets, skip-grams that make each set cohesive while distinguishing different sets are encouraged. Following this principle, we score skip-grams shared by more entity pairs from the same set higher, while penalize skip-grams shared by arbitrary pairs from different sets, as shown in the following equation.

$$
\begin{aligned}
F^{*} & =\arg \max _{|F|=Q} \frac{2}{|S| *(|S|-1)} \sum_{e_{i}, e_{j} \in S} \operatorname{Sim}\left(e_{i}, e_{j} \mid F\right) \\
& -\sum_{S_{k}, S_{k^{\prime}} \in C_{\text {aux }}} \frac{1}{\left|S_{k}\right| *\left|S_{k^{\prime}}\right|} \sum_{e_{i} \in S_{k}, e_{j} \in S_{k^{\prime}}} \operatorname{Sim}\left(e_{i}, e_{j} \mid F\right) \\
& +\sum_{S_{k} \in C_{\text {aux }}} \frac{2}{\left|S_{k}\right| *\left(\left|S_{k}\right|-1\right)} \sum_{e_{i}, e_{j} \in S_{k}} \operatorname{Sim}\left(e_{i}, e_{j} \mid F\right)
\end{aligned}
$$


where $\mathrm{Q}$ is the size of skip-grams to be selected. To solve the NPhard optimization problem in Equation (3), we apply a greedy selection process that selects the feature that increments the score the most at each time. This would benefit us in selecting complimentary features instead of focusing on strong but redundant signals.

4.3.3 Dynamically Adjusting Skip-gram Contexts. Since skipgram contexts impose hard pattern matching constraints, the candidate pool might suffer from a low coverage since some infrequent entities might not even appear in those type-indicative skip-grams. To overcome this sparsity problem, we design a transformation for skip-gram features to be more flexible, so that entities can match with skip-grams in a "softer" way.

Table 1: A frequency submatrix $\phi$ of president entities and their co-occured skip-grams.

\begin{tabular}{|c|c|c|c|c|c|}
\hline Entities & $\begin{array}{c}\text { President } \\
\text { and }\end{array}$ & $\begin{array}{c}\text { President } \\
\text { - },\end{array}$ & $\begin{array}{c}\text { President } \\
\text { - said }\end{array}$ & $\begin{array}{c}\text { President } \\
\text { 's }\end{array}$ & $\begin{array}{c}\text { President } \\
\text { * }^{-}\end{array}$ \\
\hline Bill Clinton & 33 & 17 & 2 & 23 & 75 \\
Hu Jintao & 9 & 8 & 3 & 0 & 20 \\
Gorbachev & 2 & 3 & 0 & 2 & 7 \\
\hline
\end{tabular}

In Table 1 we list a sub-matrix $\phi$ of $\Phi$ with several president entities and their co-occurred skip-grams. The infrequent "Gorbachev" does not have enough co-occurrence with type-indicative skip-grams and might be even filtered out from the candidate pool What's more, the skip-gram of "President__ said" might be neglected in the context feature selection process due to its lack of interaction with president entities. These four different skip-grams are actually similar since they can all be filled in with a president entity. Therefore, if we can extract the meaningful part from the original window size, such as "President__" in this example, we will be able to merge these different skip-grams into a common and more flexible pattern: "President __ *", which we named as a flexgram. The last term is an asteroid which serves as a wildcard term to be matched with any words. Then we can update the co-occurrence matrix by

$$
\Phi_{e, f l e x(c)}=\sum_{f l e x\left(c^{\prime}\right)=f l e x(c)} \Phi_{e, f l e x\left(c^{\prime}\right)}
$$

where flex(c) means the flexible transformation of local context $c$. Then we can merge those infrequent but type-indicative skip-grams together. However, one might say a trivial solution to this data sparsity issue is to exhaust a variety of window sizes, (e.g., $[-2,+2],[-$ $1,+1],[-1,0],[0,+1])$, as has been done by previous studies $[18,19,26]$. However, this approach very likely ends up generating many skipgrams that are too general. For example, the other half of "President _ and" is "__ and", which only contains stop words, not informative enough in implying the semantic class of the "skipped" term.

Our solution to this problem is to dynamically adjust the window size of a given fixed one, by extracting the independent unit from the the original skip-gram. We denote a skip-gram to be, $l=w_{0} w_{1} \ldots w_{s-1} \ldots w_{s+1} \ldots w_{n-1}$, where $s$ is the index of the "skipped" term. Then we can break down the skip-gram into two parts from $w_{i}$ and $w_{i+1}$. For example, a skip-gram "hospital in_has been" can be broken down in three ways: (1) $l_{\text {left }}^{1}=$ "hospital $* \ldots * *$ " and $l_{\text {right }}^{1}=$ "* in_has been"; (2) $l_{\text {left }}^{2}=$ "hospital in_ $* *$ " and $l_{\text {right }}^{2}=$ “* _ has been"; (3) $l_{\text {left }}^{3}=$ "hospital in_ has $*$ ” and $l_{\text {right }}^{3}=$ “**_ $\ldots$ been”. The bold parts are valid ones for possible flexible transformation that have non-asteroid terms connected with the skipped term.

For each possible transformation from a skip-gram to a flexgram, we consider the independence of the left and right part by looking at their pointwise mutual information.

$$
P M I\left(l_{\text {left }}^{i}, l_{\text {right }}^{i}\right)=\log \frac{P(l)}{P\left(l_{\text {left }}^{i}\right) \cdot P\left(l_{\text {right }}^{i}\right)}
$$

where $P(l)$ is the occurrence probability of skip-gram $l$ in the corpus. If the most independent pair satisfies

$$
\min _{i} P M I\left(l_{\text {left }}^{i}, l_{\text {right }}^{i}\right)<\gamma,
$$

we break the original skip-gram $l$ into $l_{\text {left }}^{i}, l_{\text {right }}^{i}$. In this example, suppose we have $l_{\text {left }}^{2}=$ "hospital in _ ** " and $l_{\text {right }}^{2}={ }^{*} *$ has been" to be the feasible breaking pair, we then look into the valid parts in $l_{\text {left }}^{i}$ and $l_{\text {right }}^{i}$, and compare their occurrences with the original skip-gram. If

$$
\frac{\sum_{e^{\prime}} \Phi_{e^{\prime}, l_{r i g h t}^{i}}}{\sum_{e^{\prime}} \Phi_{e^{\prime}, l}}>k
$$

then we can say $l_{\text {right }}^{i}$ generalizes too much from the original skipgram $l$ and prevent the transformation (as is the skip-gram “** has been" in this example), otherwise, we will allow the flexible transformation.

We perform this step of transformation for skip-gram contexts in the pre-processing part before the iterations of entity expansion, thus only requiring one-time calculation and will not harm the time efficiency.

4.3.4 Combining Different Context Features by Rank Ensemble. We use both skip-gram based features and distributed representation to measure the similarity between candidates and seed entities. These two features complement each other in that skip-gram based features require exact matching of textual patterns and impose stronger positional constraint, leading to fewer entities being matched, and embedding based features tend to capture more semantically similar terms while allowing more noise. Therefore, combining these two features improves the final result, as is shown in several previous studies $[19,26]$. For skip-gram based features, we first extract a fixed window size of $[-3,+3]$ around each entity mention in the corpus, and then transform the skip-grams dynamically into shorter flexgrams as is proposed in Section 4.3.3. After the transformation, co-occurrence between entity mentions and context features are recalculated by summing over same flexgrams. For embedding based features, we incorporate BERT, a recently introduced pre-trained language model for contextualized word representation [6], which has shown to be very effective on several NLP tasks including set expansion [26]. To get a context-free embedding for each term, we use the pre-trained model to first get the contextualized embedding of an entity mention at each occurrence, and then average over all the occurrences in the corpus to get its center point in the embedding space.

We separately score the candidates based on skip-gram and embedding features. 


$$
\begin{aligned}
& \text { score }_{s g, S}(e)=\frac{1}{|S|} \sum_{e^{\prime} \in S} \operatorname{Sim}\left(e, e^{\prime} \mid F^{*}\right) \\
& \operatorname{score}_{e m b, S}(e)=\frac{1}{|S|} \sum_{e^{\prime} \in S} \cos \left(v_{e}, v_{e^{\prime}}\right)
\end{aligned}
$$

skip-gram features based similarity is defined in Equation (2), while embedding based similarity is calculated by cosine similarity. We remove candidates if $\operatorname{score}_{S}(e)$ is smaller than any $\operatorname{score}_{S_{k}}(e)$ where $S_{k} \in C_{\text {aux }}$. We then create two pre-ranking lists of candidates based on their score from both skip-gram and embedding features, and use rank ensemble [19] to combine results from both rankings to get the final score for each candidates.

$$
\operatorname{MRR}(e)=\frac{1}{r_{s g}(e)}+\frac{1}{r_{e m b}(e)}
$$

Mean reciprocal ranking result based on the pre-ranking lists of two separate features, is used for sorting. The expansion algorithm naturally stops when all candidates have a lower similarity with seeds in target set than that with seeds in auxiliary set(s). Algorithm 2 describes the Multiple Sets Co-Expansion module.

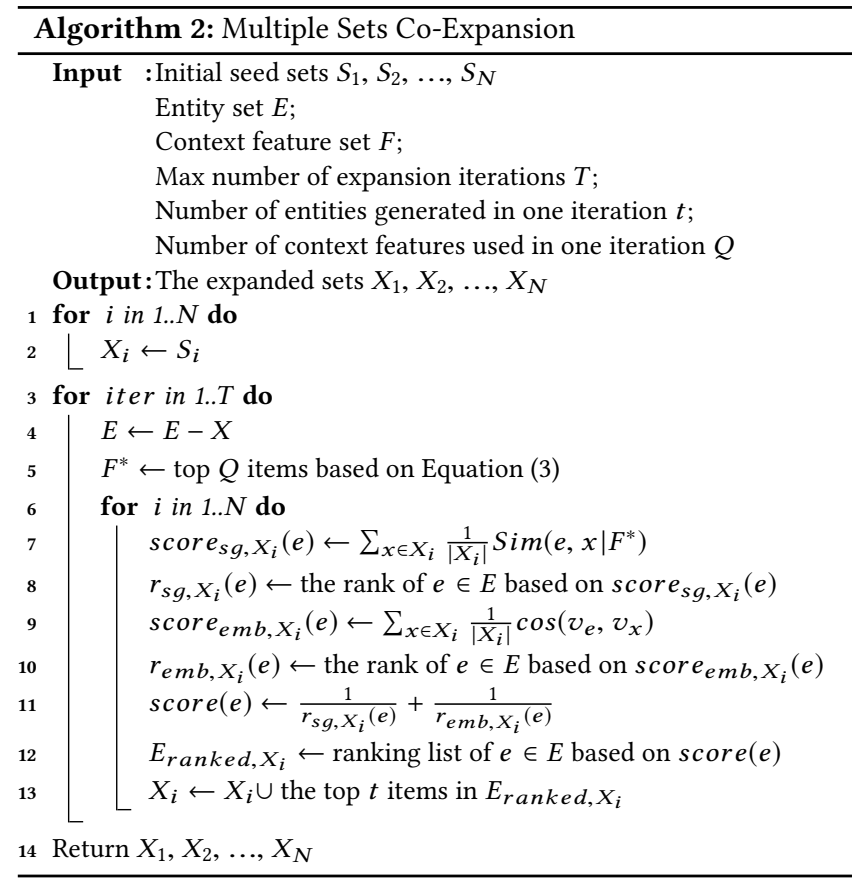

\section{EXPERIMENTS AND RESULTS}

\subsection{Experiment Setup}

\begin{tabular}{|c|c|c|c|c|}
\hline Dataset & \# classes & \# queries & entity vocabulary size & \# documents \\
\hline Wiki & 8 & 40 & 41242 & 780556 \\
\hline APR & 3 & 15 & 71707 & 1014140 \\
\hline
\end{tabular}

Table 2: Descriptions of datasets.
5.1.1 Datasets. We conduct our experiments on two large corpus also used in previous studies [19]: (1) Wiki is a subset of Wikipedia English dump from May 2011. (2) APR contains news articles published by two news platforms, Associated Press and Reuters in the year of 2015. We use the same sets of seed queries and ground truth of each semantic classes with previous studies as well. For Wiki dataset, there are 8 semantic classes, and 5 queries per class. For APR dataset, there are 3 semantic classes, and 5 queries for each class. These queries cover a wide range of semantic classes. Each query contains three seed terms belonging to a certain semantic class. Table 2 lists the detailed descriptions of these datasets and queries ${ }^{1}$.

To extract possible entity mentions, we use the same preprocessing pipeline as SetExpan [19].

5.1.2 Parameter Settings. Since our framework iteratively generates auxiliary sets and co-expands target and auxiliary sets, we set the expansion number $t$ at each iteration to be 5 , and will later provide parameter study of $t$. In the auxiliary sets generation module, the embedding training balances the global context loss and local context loss by a ratio $\lambda$ of 1.5 , and we retrieve 10 related words for each element in the current expanded set. In the cross-seed merging stage, two groups can be merged together if they have enough overlap, thus we set $\eta$ to be $\sqrt{\min \left(g_{e, \text { expanded }}^{i}, g_{\text {e,expanded }}^{j}\right)}$. In the multiple sets co-expansion module, since the size of generated auxiliary sets are often larger than the currently expanded target set, we need to balance the size of multiple sets to prevent the model from extracting features that only focus on making the largest set to be cohesive. We randomly sample seeds from each auxiliary set to keep it as large as the target set. When extracting skip-gram features for each entity mention, we first extract a fixed window size of 3, and then transform them into dynamic independent units as described in section 4.3.3, and there are two parameters in this transformation: the breaking threshold $\gamma$ is set to be 1.0 , and the generalizing threshold $k$ is set to be 100 , which stops the original skip-gram to be broken down to too general contexts. In the Algorithm 2, we set $T=10$ and $Q=200$ as SetExpan [19]. We use the same hyperparameters for all queries on both datasets.

5.1.3 Evaluation Metric. We use Mean Average Precision (MAP) to evaluate the ranking result of our methods as well as other baseline methods. For a query $q$ with 3 seeds, we take the first $k$ output entities from each algorithm as list $L$ and calculate Average Precision at $k=10,20,50$, defined as

$$
A P_{k}(L)=\sum_{i=1}^{k} P(k) \Delta r(k)
$$

where $P(k)$ denotes precision@ $k$ and $\Delta r(k)$ denotes the change in recall from the $(k-1)^{t h}$ term to the $k^{t h}$ term. Therefore, only the terms expanded correctly will contribute to this metric. Mean Average Precision at $k$ is defined as the mean of Average Precision at $k$ over all queries in all semantic classes.

$$
M A P_{k}=\frac{\sum_{L} A P_{k}(L)}{|L|}
$$

\footnotetext{
${ }^{1}$ The code and data are available at https://github.com/teapot123/SetCoExpan
} 
Table 3: Mean Average Precision across all queries on Wiki and APR.

\begin{tabular}{|c|c|c|c|c|c|c|}
\hline Methods & MAP@10 & $\begin{array}{c}\text { Wiki } \\
\text { MAP@20 }\end{array}$ & MAP@50 & MAP@10 & $\begin{array}{c}A P R \\
\text { MAP@20 }\end{array}$ & MAP@50 \\
\hline CaSE & 0.897 & 0.806 & 0.588 & 0.619 & 0.494 & 0.330 \\
\hline SetExpander & 0.499 & 0.439 & 0.321 & 0.287 & 0.208 & 0.120 \\
\hline SetExpan & 0.944 & 0.921 & 0.720 & 0.789 & 0.763 & 0.639 \\
\hline BERT & 0.970 & 0.945 & 0.853 & 0.890 & 0.896 & 0.777 \\
\hline Set-CoExpan (no aux.) & 0.964 & 0.950 & 0.861 & 0.900 & 0.893 & 0.793 \\
\hline Set-CoExpan (no flex.) & 0.973 & 0.961 & 0.886 & 0.927 & 0.908 & 0.823 \\
\hline Set-CoExpan & 0.976 & 0.964 & 0.905 & 0.933 & 0.915 & 0.830 \\
\hline
\end{tabular}

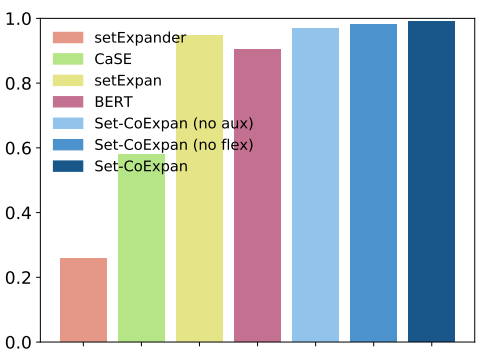

(a) Wiki: countries

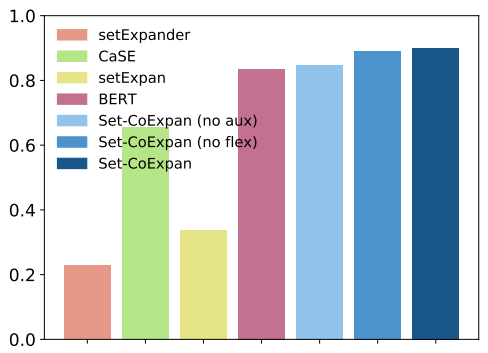

(d) Wiki: TV channels

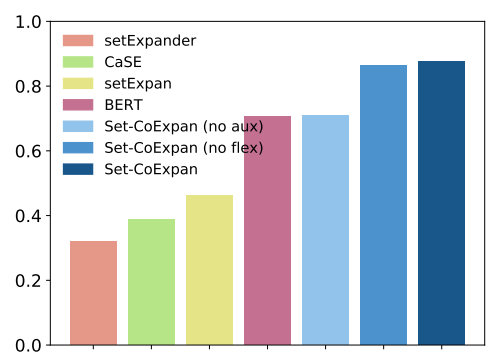

(b) Wiki: companies

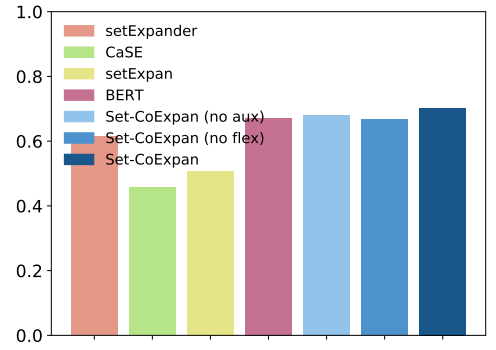

(e) Wiki: china provinces

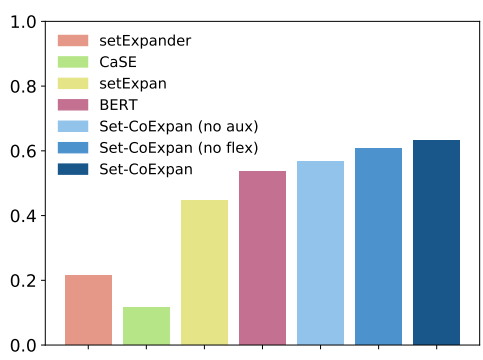

(c) APR: Political Parties

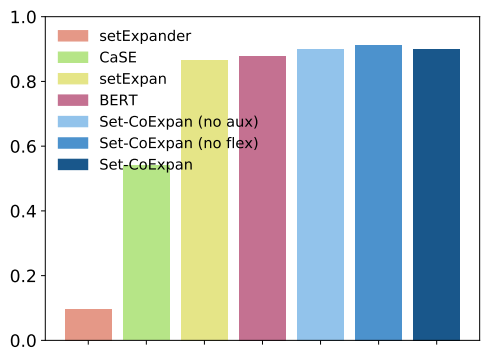

(f) APR: US states

Figure 4: Performance comparison on different semantic classes in Wiki and APR.

\subsection{Compared Methods}

We compare our methods to several previous corpus-based set expansion algorithms that only take corpus as input to expand entity seed sets. We also implement a baseline named BERT that only uses the pre-trained BERT model to retrieve the most similar entities. To show the effectiveness of auxiliary sets and flexible transformation of skip-grams in improving set expansion, we also compared Set-CoExpan with several ablations.

- SetExpan [19]: This method uses skip-gram features, distributed representations and coarse-grained types as context features. It uses rank ensemble to combine the result of three pre-ranking lists. We run the algorithm using its default setting.

- SetExpander [13]: This method incorporates multiple context features by training separate embedding space for each type of contexts, and a classifier is trained on a labeled dataset to adjust the weight of different features.
- CaSE [26]: This method combines skip-gram patterns and distributed representations to generate a one-time ranking for candidate entities. The model has three variants using different distributed representations, and we report the result of CaSE-W2V which has the highest performance.

- BERT [6]: We implement a baseline that only uses BERT, a recent contextualized embedding framework. We use the pre-trained model (uncased, base, 768 dimensions) and average over all occurrences in the corpus to get the centroid for any entity. We then use KNN to search for most similar entities.

- Set-CoExpan (no aux.): This ablation excludes the auxiliary sets generation module thus only expand one query of seed set in the co-expansion module.

- Set-CoExpan (no flex.): This ablation expands auxiliary sets simultaneously with the target set. However, it removes the flexible transformation of skip-grams. 
Table 4: Auxiliary sets generated for various queries.

\begin{tabular}{|c|c|c|}
\hline Class & Query & Auxiliary sets \\
\hline Companies & Myspace, Youtube, Twitter & (Products): flickr, wordpress, google earth, gmail, google maps \\
\hline Countries & Australia, France, Germany & $\begin{array}{l}\text { (Provinces): Queensland, New South Wales, Saxony, Bavaria, Thuringia } \\
\text { (Cities): Brisbane, Canberra, Rennes, Hamburg, Stuttgart }\end{array}$ \\
\hline TV Channels & ESPN News, ESPN Classic, ABC & $\begin{array}{l}\text { (TV Programmes): the young and the restless, } \\
\text { all my children, guiding light, general hospitale }\end{array}$ \\
\hline Sports Leagues & $\begin{array}{c}\text { national football league, } \\
\text { national hockey league, } \\
\text { major league baseball }\end{array}$ & $\begin{array}{l}\text { (Sports Teams): new york jets, ottawa senators, } \\
\text { chicago white sox, dallas cowboy, st.louis hawks }\end{array}$ \\
\hline Political Parties & $\begin{array}{c}\text { new democratic party, } \\
\text { liberal party of canada, } \\
\text { northern ireland labour party }\end{array}$ & $\begin{array}{l}\text { (Elections): } 1980 \text { federal election, } 1997 \text { federal election, } 1980 \text { election, } \\
1962 \text { election, } 2008 \text { provincial election }\end{array}$ \\
\hline Chinese Provinces & jiangsu, liaoning, sichuan & (China Cities): xi'an, hangzhou, shanghai, chengdu, beijing \\
\hline Diseases & $\begin{array}{l}\text { tuberculosis, } \\
\text { parkinson's disease, } \\
\text { esophageal cancer }\end{array}$ & (Symptoms): tumor, dehydration, dementia, muscle stiffness \\
\hline US States & Texas, Florida, New Mexico & (US Cities): fort worth, san antonio, jacksonville, tampa, orlando \\
\hline
\end{tabular}

\subsection{Results and Discussions}

5.3.1 Overall Performance on Two Datasets. For our method and all baseline methods, we use the same queries in the datasets, and report MAP@k $(k=10,20,50)$ in Table 3 . The result clearly shows that our model has the best performance over all the methods in both datasets. Among all the baseline methods, BERT is the strongest one, since it stores a very large pre-trained language model to represent word semantics in a piece of context, which also explains why we have a very large margin over previous iterative-based methods like SetExpan when we incorporate BERT as embedding-based features. However, BERT itself could not outperform our model, which implies that modeling distributional similarity alone is not enough for generating accurate expansion. We can also observe that Set-CoExpan also outperforms the ablations without auxiliary sets or flexgram, which justifies that auxiliary sets indeed improve the set expansion process by providing clearer discriminative context features for multiple sets to prevent them from expanding into each other's semantic territory. The advantage of Set-CoExpan over the ablation without flexgram also proves that it is necessary to cope with the data sparsity problem by extracting independent units from a fixed local context window. In this way, we relieve the hard-matching constraint brought by skip-gram features by making it more flexible. What's more, Set-CoExpan has a larger advantage over all the baselines when the ranking list is longer, which indicates that when the seed set gradually grows out of control and more noises appear, our method is able to steer the direction of expansion and to set barriers for out-of-category words to come in.

5.3.2 Performance Comparison on Different Semantic Classes. We further break down the results on different semantic classes to see which method achieves the best performance on each semantic class, as shown in Figure 4. We can see that the performances of several baseline methods can be rather unstable over different semantic classes, while our method can outperform them in all cases. For example, setExpander gives good results in China Provinces but bad results for other classes. This is because the weights in

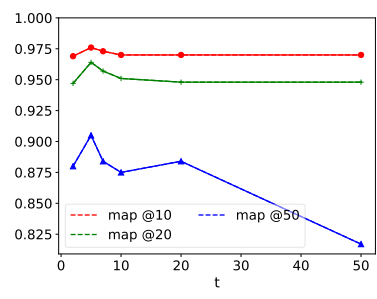

(a) Wiki

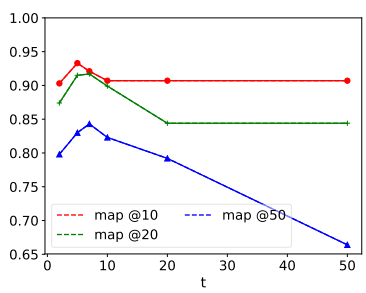

(b) APR
Figure 5: Parameter Study.

its classifier is pre-trained on other corpus with supervision, and might not adapt well to copora without labels for training. setExpan shows promising results in Countries and US States, but still suffers from the problem of semantic drift in other categories.

The margin between Set-CoExpan and the two ablations also varies between semantic classes. We can observe that for classes like "Countries", "TV Channels", "Political Parties" and "Companies", the auxiliary sets help improve the performance to a great extent. On the other hand, for some categories like "China Provinces" and "US States", the improvement is marginal, which implies that in these situations our method does not generate meaningful auxiliary sets to for the target set to compare with. Since our method of generating auxiliary sets is entirely unsupervised, it is not guaranteed that each semantic class can find very meaningful auxiliary sets.

\subsection{Parameter Study}

Since our model expands $t$ entities into the seed set iteratively, we are interested in how the number of entities expanded at each iteration affects the overall performance of the final output. We vary $t$ from 2 to 50 and plot MAP@K $(K=10,20,50)$ on both Wiki and $A P R$ dataset in Figure 5. We observe that the optimal settings for both Wiki and APR dataset is around $t=5$. The reason is that on one hand, when the number of newly added entities in one iteration is too small, a single noisy term can create a big impact which results in semantic drift. On the other hand, when $t$ is too 
Table 5: Results of Co-Expansion and Separate Expansion of Target Set and Auxiliary Sets.

\begin{tabular}{|c|cc|cc|cc|}
\hline \multirow{2}{*}{ seeds } & \multicolumn{2}{|c|}{ seeds from Target Set: } & \multicolumn{2}{c|}{ seeds from Aux. Set 1: } & \multicolumn{2}{c|}{ seeds from Aux. Set 2: } \\
& Australia, France, Germany & Queensland, Saxony, New South Wales & Brisbane, Canberra, Stuttgart \\
\hline & Italy & Luxembourg & Baden-Wurttemberg & Hesse & Berlin & Hanover \\
Multiple Sets & Canada & Belgium & Baden & Saxony-Anhalt & Dortmund & Frankfurt \\
Co-Expansion & Norway & Spain & Schleswig-Holstein & Silesia $(\boldsymbol{X})$ & Heidelberg & Strasbourg \\
& The Netherlands & Denmark & Rhineland-Palatinate & WestPhalia & Munich & Bonn \\
& England & Switzerland & Mecklenburg-Vorpommern & Saarland & Cologne & Mannheim \\
\hline \multirow{3}{*}{ Separate } & Italy & Luxembourg & Baden-Wurttemberg & WestPhalia & Strasbourg & Berlin \\
Expansion & Canada & Belgium & Hesse & Saxony-Anhalt & Marseille & Hanover \\
of Each Set & Spain & Brussels $(\boldsymbol{x})$ & Baden & Berlin & Auxerre & Lyon \\
& England & Paris $(\boldsymbol{x})$ & Wurttemberg & Munich $(\boldsymbol{x})$ & AS Saint-Etienne $(\boldsymbol{x})$ & Nancy \\
& Switzerland & Ireland & Franconia $(\boldsymbol{x})$ & Stuttgart $(\boldsymbol{x})$ & Paris Saint-Germain $(\boldsymbol{x})$ & Lens \\
\hline
\end{tabular}

large, the model benefits minimally from the iterative design since the feature pool is not updated timely.

\subsection{Case Study}

5.5.1 Qualitative Analysis of Generated Auxiliary Sets. We are interested in what kind of auxiliary classes would be generated for different semantic classes. In Table 4 we showcase one auxiliary sets generation result for each semantic class using real queries from the Wiki dataset. It is amazing that in most cases, our method can extract meaningful related semantic classes with respect to the target class. For example, a set of provinces and cities are generated for the category of countries. This explains why our model can avoid to wrongly expand more fine-grained location names by comparing them to countries. For the category of Sports Leagues, the sports teams affiliated to different sports leagues are extracted, and sports teams can share very similar local context features with sports leagues, such as "best player in _.". We can also find Symptoms as a new auxiliary set corresponding to the "Diseases" set. By comparing the difference in their skip-gram features, our multiple sets coexpansion algorithm can distinguish these two classes apart. The high quality and diversity of auxiliary sets and their relations with the core set shows that our framework can be generalized to many other semantic classes.

5.5.2 Effectiveness of Multiple Sets Co-Expansion. To justify the effectiveness of our multiple sets co-expansion module which takes the auxiliary sets as input to help guide the target set expansion, we randomly select two semantic classes in Wiki Dataset and show how the expansion of both target set and auxiliary sets benefit from contrastive feature selection. In Table 5 we list 10 entities expanded at the first two iterations using Set-CoExpan or Set-CoExpan (no aux.) which is the same as expanding each set separately. Terms that should not be expanded (since they are in a different category) are marked with $(\boldsymbol{X})$. The result proves the effectiveness of our multiple sets co-expansion module that chooses the most discriminative local context features by comparing seeds from different semantic classes. Specifically, for the query \{Australia, France, Germany\} that belongs to Countries, without guidance of auxiliary sets, the original expansion process can be confused by entities from classes of different granularities (e.g., Provinces and Cities), as is shown in the table that some cities such as Brussels and Paris, are expanded. However, the auxiliary sets generated by our model as shown in both Table 4 and Table 5 are sets of provinces and cities that are located in the seed countries: \{Queensland, New South Wales, Saxony\} and \{Brisbane, Canberra, Stuttgart\}. After scoring the context features by their ability to discriminate different semantic classes, the expansion results of both the target class and the auxiliary sets involve fewer cross-class errors, verifying that the multiple sets co-expansion module can effectively utilize the information provided by auxiliary sets.

\section{CONCLUSION AND FUTURE WORK}

In this paper we explore the problem of semantic drift in single query set expansion. Our proposed framework Set-CoExpan generates auxiliary sets for current expanded results and co-expands the target set and auxiliary sets in an iterative manner. The auxiliary sets generation module retrieves related terms, and then merge the terms by their relations to the expanded seeds, forming related but different semantic classes. The multiple sets co-expansion module later takes both target set and auxiliary sets into consideration and co-expands them by extracting the most discriminative context features. Extensive experiments demonstrate that Set-CoExpan has a good generalizability by capturing high quality auxiliary sets as rival sets for various queries, and these sets do help guide the expansion of target set to avoid falling into pitfalls of related but different semantic types.

For future work, it is interesting to study how we can leverage auxiliary sets to form different semantic classes in the distributed space, so that not only skip-gram features but also embedding features can be used to discriminate them. Our paper only focuses on named entity expansion, while the problem of expanding generalized nouns (i.e., concepts) is more challenging and worth exploring.

\section{ACKNOWLEDGMENTS}

Research was sponsored in part by DARPA under Agreements No. W911NF-17-C-0099 and FA8750-19-2-1004, National Science Foundation IIS 16-18481, IIS 17-04532, and IIS-17-41317, and DTRA HDTRA11810026. Any opinions, findings, and conclusions or recommendations expressed in this document are those of the author(s) and should not be interpreted as the views of any U.S. Government. The U.S. Government is authorized to reproduce and distribute reprints for Government purposes notwithstanding any copyright notation hereon. We thank anonymous reviewers for valuable and insightful feedback. 


\section{REFERENCES}

[1] Carl Allen and Timothy M. Hospedales. 2019. Analogies Explained: Towards Understanding Word Embeddings. In ICML.

[2] Maurizio Atzori, Simone Balloccu, and Andrea Bellanti. 2018. Unsupervised Singleton Expansion from Free Text. In ICSC.

[3] Antoine Bordes, Nicolas Usunier, Alberto García-Durán, Jason Weston, and Oksana Yakhnenko. 2013. Translating Embeddings for Modeling Multi-relational Data. In NIPS

[4] Huanhuan Cao, Daxin Jiang, Jian Pei, Qi He, Zhen Liao, Enhong Chen, and Hang Li. 2008. Context-aware query suggestion by mining click-through and session data. In $K D D$.

[5] Zhe Chen, Michael J. Cafarella, and H. V. Jagadish. 2016. Long-tail Vocabulary Dictionary Extraction from the Web. In WSDM.

[6] Jacob Devlin, Ming-Wei Chang, Kenton Lee, and Kristina Toutanova. 2019. BERT: Pre-training of Deep Bidirectional Transformers for Language Understanding. In NAACL-HLT.

[7] Oren Etzioni, Michael J. Cafarella, Doug Downey, Ana-Maria Popescu, Tal Shaked, Stephen Soderland, Daniel S. Weld, and Alexander Yates. 2005. Unsupervised named-entity extraction from the Web: An experimental study. Artif. Intell. 165 (2005), 91-134.

[8] Sonal Gupta, Diana L. MacLean, Jeffrey Heer, and Christopher D. Manning. 2014. Research and applications: Induced lexico-syntactic patterns improve information extraction from online medical forums. Fournal of the American Medical Informatics Association : 7AMIA 215 (2014), 902-9.

[9] Sonal Gupta and Christopher D. Manning. 2014. Improved Pattern Learning for Bootstrapped Entity Extraction. In CoNLL

[10] Prateek Jindal and Dan Roth. 2011. Learning from Negative Examples in SetExpansion. 2011 IEEE 11th International Conference on Data Mining (2011), 11101115

[11] Winston Lin, Roman Yangarber, and Ralph Grishman. 2003. Bootstrapped learning of semantic classes from positive and negative examples.

[12] Yankai Lin, Zhiyuan Liu, Maosong Sun, Yang Liu, and Xuan Zhu. 2015. Learning Entity and Relation Embeddings for Knowledge Graph Completion. In AAAI.

[13] Jonathan Mamou, Oren Pereg, Moshe Wasserblat, Alon Eirew, Yael Green, Shira Guskin, Peter Izsak, and Daniel Korat. 2018. Term Set Expansion based NLP
Architect by Intel AI Lab. In EMNLP.

[14] Yu Meng, Jiaxin Huang, Guangyuan Wang, Zihan Wang, Chao Zhang, Yu Zhang, and Jiawei Han. 2020. Discriminative Topic Mining via Category-Name Guided Text Embedding. In $W W W$.

[15] Tomas Mikolov, Ilya Sutskever, Kai Chen, Gregory S. Corrado, and Jeffrey Dean. 2013. Distributed Representations of Words and Phrases and their Compositionality. In NIPS.

[16] John M. Prager, Jennifer Chu-Carroll, and Krzysztof Czuba. 2004. Question Answering Using Constraint Satisfaction: QA-By-Dossier-With-Contraints. In ACL.

[17] Lior Rokach and Oded Maimon. 2005. Clustering Methods. In The Data Mining and Knowledge Discovery Handbook

[18] Xin Rong, Zhe Chen, Qiaozhu Mei, and Eytan Adar. 2016. EgoSet: Exploiting Word Ego-networks and User-generated Ontology for Multifaceted Set Expansion. In WSDM.

[19] Jiaming Shen, Zeqiu Wu, Dongming Lei, Jingbo Shang, Xiang Ren, and Jiawei Han. 2017. SetExpan: Corpus-Based Set Expansion via Context Feature Selection and Rank Ensemble. In ECML/PKDD.

[20] Michael Thelen and Ellen Riloff. 2002. A Bootstrapping Method for Learning Semantic Lexicons using Extraction Pattern Contexts. In EMNLP.

[21] S. Tong and J. Dean. 2008. System and methods for automatically creating lists. US Patent 7,350,187.

[22] Paola Velardi, Stefano Faralli, and Roberto Navigli. 2013. OntoLearn Reloaded: A Graph-Based Algorithm for Taxonomy Induction. Computational Linguistics 39 (2013), 665-707.

[23] Vishnu Vyas and Patrick Pantel. 2009. Semi-Automatic Entity Set Refinement. In HLT-NAACL

[24] Richard C. Wang, Nico Schlaefer, William W. Cohen, and Eric Nyberg. 2008. Automatic Set Expansion for List Question Answering. In EMNLP.

[25] Zhen Wang, Jianwen Zhang, Jianlin Feng, and Zhigang Chen. 2014. Knowledge Graph Embedding by Translating on Hyperplanes. In AAAI.

[26] Puxuan Yu, Zhiqi Huang, Razieh Rahimi, and James Allan. 2019. Corpus-based Set Expansion with Lexical Features and Distributed Representations. In SIGIR.

[27] Wanzheng Zhu, Hongyu Gong, Jiaming Shen, Chao Zhang, Jingbo Shang, Suma Bhat, and Jiawei Han. 2019. FUSE: Multi-Faceted Set Expansion by Coherent Clustering of Skip-grams. ArXiv abs/1910.04345 (2019). 